\title{
Valentina Di Rosa \\ „In der Situation des Radwechslers“: Ingeborg Bachmanns Berliner Periode als Öffnung zu einer transnationalen Literaturpraxis
}

\author{
Und der verändern wollende Dichter, \\ wieviel steht ihm frei und wieviel nicht? ${ }^{1}$
}

\section{Einleitung: Unterwegs zu einer transnationalen ,Repräsentanz ${ }^{\circ}$}

Versucht man Ingeborg Bachmanns Positionierung auf der geopolitischen Landkarte des geteilten Europas der frühen 1960er Jahre nachzuzeichnen, so ergeben sich gleich drei Trajekte und ein Ausgangspunkt. Als solcher gilt die Stadt Berlin, genauer gesagt: die spezielle Enklave West-Berlin, wo sich die Dichterin vom Frühjahr 1963 bis Ende 1965 zunächst als Stipendiatin der Ford Foundation aufhält. Es sind insgesamt fast drei Jahre, die im unmittelbaren Zeichen des Mauerbaus und der konsequenten Zuspitzung des Ost-West-Konflikts stehen und die auch für Bachmanns literaturpolitische Praxis nicht ohne Folgen bleiben:

Daß Dichten außerhalb der geschichtlichen Situation stattfindet, wird wohl heute niemand mehr glauben - daß es auch nur einen Dichter gibt, dessen Ausgangsposition nicht von den Zeitgegebenheiten bestimmt wird. Gelingen kann ihm, im glücklichsten Fall, zweierlei: zu repräsentieren, seine Zeit zu repräsentieren und etwas zu repräsentieren, für das die Zeit noch nicht gekommen ist. (KS, 266-267)

Hatten bereits die Frankfurter Vorlesungen (1959/60) ihr Verständnis von Zeitgenossenschaft nachdrücklich pointiert, so betrachtet nun Bachmann die Eskalation der

1 Ingeborg Bachmann: Frankfurter Vorlesungen. Probleme zeitgenössischer Dichtung, in: dies.: Werke, hrsg. von Christine Koschel und Inge von Weidenbaum, 4 Bde., München/Zürich 1978, Bd. 4, 182-271, hier: 197. (Im Folgenden werden Zitate aus dieser Edition mit der Sigle W sowie mit Band- und Seitenangabe direkt im Text nachgewiesen). Vgl. auch dies.: Kritische Schriften, hrsg. von Monika Albrecht und Dirk Göttsche, München/Zürich 2005, 268. (Im Folgenden werden Zitate aus dieser Edition mit der Sigle KS sowie mit Seitenangabe direkt im Text nachgewiesen). 
Berlin-Krise als Movens zu einer gebotenen „Radikalisierung“² ihrer öffentlichen Stimme - und zwar eben in zweierlei Hinsicht: Auf der einen Seite drängt die tagespolitische Aktualität zu einer schonungslosen Diagnose des „Virus Europa“ (KS, 392); auf der anderen gewinnt die Projektion auf einen ästhetisch-politischen Horizont der Transnationalität bzw. Transkulturalität als Kontrapunkt zur Spaltung des Kontinents einen eigenen, zunehmend gewichtigen Raum.

So führt das erste Trajekt im April 1963 in Zusammenhang mit dem Plan einer neu zu gründenden Revue Internationale nach Paris. Hervorgegangen war die Initiative zwei Jahre zuvor aus einem heterogenen Ensemble von Intellektuellen aus Frankreich, Deutschland und Italien, mit dem Ziel, im Spannungsfeld des Kalten Krieges einen neuen, länderübergreifenden Handlungsraum für die Literatur als Medium der Zeitkritik zu schaffen.

Im Nachhinein wird sich das Pariser Redaktionstreffen freilich als Auftakt zu einer endgültigen Auflösung der Gruppe erweisen. Als Kompromisslösung wird im März 1964 unter dem Titel Gulliver eine einzige sogenannte ,Nullnummer der geplanten Zeitschrift in Italien gedruckt, die relativ schnell als Bestätigung eines kollektiven Scheiterns ad acta gelegt werden wird. Davon, dass sich Bachmann zumal in der programmatischen Vorbereitungsphase an der vorderen Front engagiert, zeugt nicht zuletzt die Publikation ihres Diario in pubblico ${ }^{3}$ - ein bis dato relativ wenig beachteter Essay, in dem sie zwischen Analyse und Vision eine entmystifizierende Lektüre der herrschenden Propaganda-Diskurse im geteilten Europa der Zeit artikuliert und zugleich eine eigene Bilanz über das pionierhafte Experiment jenes transnationalen Gegenentwurfs zieht.

Das zweite Trajekt führt Bachmann im Oktober 1964 nach Darmstadt anlässlich der Entgegennahme des Georg-Büchner-Preises. Aus der extraterritorialen Perspektive West-Berlins gestaltet sich eine solche Reise nicht nur wegen der ideologischen Distanz von den restaurativen Tendenzen der Bundesrepublik der Adenauerära als eine Reise nachgerade in die Fremde.

In diesem Sinn bietet sich in ihren Augen der fest ritualisierte Akt der Dankesrede als passende Gelegenheit, das institutionelle Auditorium mit einer frontalen Abrechnung mit der tabuisierten „Beschädigung“ Berlins (TKA I, 190) zu konfrontieren, die hier als „Konsequenz auf die Konsequenz“ (ebd.) in enger Verkettung mit der unverarbeiteten Nazivergangenheit offen thematisiert wird. Indem sie sich in der Rolle eines außenstehenden „Ortsfremde[n]“ (TKA I, 232) programmatisch zu erkennen gibt,

2 Ingeborg Bachmann: „Todesarten“-Projekt. Kritische Ausgabe, hrsg. von Monika Albrecht und Dirk Göttsche unter Leitung von Robert Pichl, 5 Bde., München/Zürich 1995, Bd. 1, 232 (Im Folgenden werden Zitate aus dieser Edition mit der Sigle TKA sowie mit Band- und Seitenangabe direkt im Text nachgewiesen).

3 Ingeborg Bachmann: Diario in pubblico, in: Gulliver / il menabò. Una rivista internazionale 7 (1964), 262-274 (für die deutsche Originalfassung vgl. KS, 378-395). 
fokussiert die Dichterin mit entsprechend unbequemem Gestus die Mauerstadt als einen Ort für „Zufälle“ beziehungsweise als neuralgischen Schauplatz einer kollektiven Verdrängung, um dann im Hier und Jetzt ihrer Ansprache gleichsam eine Wiederkehr des Verdrängten literarisch zu inszenieren. Leitendes Credo: „Die Wahrheit ist dem Menschen zumutbar“. (KS, 246-248)

Das dritte Trajekt bezieht sich auf die Reise, die Bachmann zwischen Januar und März 1964 jenseits des Eisernen Vorhangs nach Prag unternimmt - eine ,Winterreise“ in die ehemalige Tschechoslowakische Sozialistische Republik, wo sich ein neues Klima der ideologischen Lockerung als Auftakt zum kurzen ,Frühling، der politischen Reformen bereits ankündigte und die Stadt Prag als solche nach Meinung vieler internationaler Beobachter noch einen der hoffnungsträchtigen Orte Europas repräsentierte.

Diese Reise führt Bachmann zugleich in eine verlorene Welt von affektiven Erinnerungsspuren und literarischen Resonanzen, die in direkter Relation mit der Entstehung eines ihrer berühmtesten Gedichte steht: Böhmen liegt am Meer. Seit Shakespeares Winter's Tale wandert bekanntlich das prominente Phantasiebild als mächtige Suggestion im kulturellen Gedächtnisarchiv Europas und Bachmann nimmt es hier gerne als Motiv auf, um eine eigene, subjektiv gefärbte und zugleich politisch konnotierte mitteleuropäische Kartographie zu entwerfen.

Ist die Rede in Darmstadt gleichsam als ,Export‘ einer unheimlichen BerlinDystopie nach Westdeutschland intendiert, so entfaltet das Böhmen-Gedicht die Implikationen einer umgekehrten Denkbewegung, nämlich die versöhnende Imagination eines poetisch-politischen Territoriums, wo ein friedliches, austauschorientiertes Miteinander von Sprachen, Kulturen, Identitäten in utopischer Gestalt Konturen gewinnt.

Hier wird bereits eine gedankliche Nähe zum Programm der Revue erkennbar, wobei der transnationale Charakter des Zeitschriftenprojekts an sich einen besonders aufschlussreichen Zusammenhang darstellt, um Bachmanns Engagement der Berliner Jahre näher zu beleuchten. Ein roter Faden durchzieht nämlich die drei nur scheinbar disparaten Interventionsfelder. Ein ,Tagebuch', eine Rede, ein Gedicht: In allen drei Fällen meldet sich Bachmann als österreichische Staatsbürgerin und zugleich deutschsprachige Autorin an der Schnittstelle zwischen nationalen und transnationalen Diskursordnungen zu Wort, indem sie die weltpolitische Konstellation ihrer Gegenwart direkt adressiert und dabei eng korrelierte literaturpolitische beziehungsweise literaturästhetische Kategorien ansetzt: Berlin und Europa, Deutschland und Mitteleuropa sowie, obzwar zwischen den Zeilen, Österreich. 


\section{„Verlaufen auf diesem Planeten“: Zu Gast in der geteilten Stadt}

Als gebürtige Kärntnerin wohnt Bachmann zum Zeitpunkt des Berliner Stipendiums nach mehreren Reisen und provisorischen Aufenthalten - u.a. in Wien, Paris, München, New York, Neapel, Ischia - zwischen Rom und Zürich. ${ }^{4}$

Öfters im Lauf der darauffolgenden Jahre wird sie ein solches „Vagabunden“Dasein oder gar „Doppelleben“ ${ }^{\text {5 }}$ als literarisch bedingte Strategie thematisieren, die es ihr ermögliche, den nötigen Abstand von ihrem Herkunftsland zu wahren. Denn mit Österreich, zumal mit der Hauptstadt Wien, unterhalte sie von Jugend auf ein höchst ambivalentes Verhältnis. Sie nennt es symbolisch „Exil“ (W I, 153), gelegentlich „Haßliebe“ (GuI, 106), wodurch ein subtil verwobenes Geflecht von literarischen Motiven und Phantasmen mobilisiert wird. Dazu gehören nicht nur die traumatischen Erfahrungen des Nationalsozialismus und des Krieges, sondern auch die aktualitätsbezogenen Reflexe einer solch rezenten Vergangenheit: die Last der Schuldfrage, die kollektiven Tabuisierungs- und Verdrängungsmechanismen, das Bewusstsein über die latente Kontinuität des Faschismus in der Gegenwart der Nachkriegszeit.

Bekanntlich repräsentiert für Bachmann der Einmarsch der Hitlertruppen in Klagenfurt eine markante biographische Zäsur, ${ }^{6}$ die sie, wie die meisten Literaten der ,Auschwitz-Generation', als moralisch verpflichtenden Ausgangspunkt ihrer Autorschaft versteht - darin liegt die Matrix einiger ihrer prägnantesten Gedichtzeilen, die im restaurativen Klima der 1950er Jahre widerhallen: „Der Krieg wird nicht mehr erklärt, / sondern fortgesetzt [...]“; „Sieben Jahre später, / in einem Totenhaus, / trinken die Henker von gestern / den goldenen Becher aus“. (W I, 44 und 46)

Mittlerweile, im Alter von 37 Jahren, ist Bachmann als erste Stipendiatin der Ford Stiftung eine durchaus renommierte, mehrfach preisgekrönte Autorin, die bereits fast am Höhepunkt ihrer literarischen Karriere angelangt ist. Ihrer steigenden Reputation entspricht eine steigende Popularität im Medienpanorama, die sich spätestens, seit ihr im Sommer 1954 das Titelbild des Spiegel gewidmet wurde, weit über die Kreise des Literaturbetriebs erstreckt.

4 „Verlaufen auf diesem Planeten“ in: Ingeborg Bachmann: „Ich weiß keine bessere Welt“. Unveröffentlichte Gedichte, hrsg. von Isolde Moser, Heinz Bachmann, Christian Moser, München/Zürich 2000, 131.

5 Ingeborg Bachmann: Wir müssen wahre Sätze finden. Gespräche und Interviews, hrsg. von Christine Koschel und Inge von Weidenbaum, München 1983, hier: 39 und 65. (Im Folgenden werden Zitate aus dieser Edition mit der Sigle GuI sowie mit Seitenangabe direkt im Text nachgewiesen).

6 Zur literarischen (Re-)Konstruktion dieser „Urszene“ vgl. u. a. Sigrid Weigel: Ingeborg Bachmann. Hinterlassenschaften unter Wahrung des Briefgeheimnisses, Wien 1999, 317; Monika Albrecht: Nationalsozialismus, in: Monika Albrecht, Dirk Göttsche (Hrsg.): Bachmann-Handbuch. Leben - Werk Wirkung, Stuttgart, Weimar 2020, 269-278. 
Das Porträt der „deutschen“ Dichterin als junge Frau in Rom ist ein vielzitiertes zeittypisches Dokument, das sich unter anderem als Beitrag zur Aufwertung des geistigen Klimas der BRD nach 1945 liest. Es ist in diesem Kontext, dass das existentialistisch anmutende, berühmt-berüchtigte Bild des „möblierte[n] Mädchen[s]“ geprägt wird - allerdings nicht ohne männliches Pendant, denn im Sinne der distinktiven „Unbehaustheit“ einer ganzen Generation von „arme[n]“ Poet:innen „in der Dachkammer" ist noch parallel die Rede, obgleich in subordinierter Stellung, vom Dichterkollegen (und "unbeamtete[n] Universitäts-Dozent“) Walter Höllerer, der ein ebenso „ruhearmes Untermieter-Dasein“ führe. ${ }^{7}$

Was abseits solch geradezu karikaturistischer Skizzierungen Bachmann und Höllerer seit ihrer ersten Begegnung bei der Gruppe 47 tatsächlich mit einander verbindet, ist neben der römischen Familiarität eine freundliche, in siezender Form gehaltene Briefkorrespondenz, in der neben spielerischen Anreden und augenzwinkernden Bemerkungen (nicht zuletzt zu den Bedingungen für „,behauste Lyrik ${ }^{\text {( } 8}$ ) ästhetische Affinitäten und gemeinsame Projekte besprochen werden.

Was seine eigene Karriere und seinen Wohnsitz anbelangt, wird Höllerer 1959 nach einer akademischen Assistenz in Frankfurt am Main als Germanistikprofessor an die Technische Universität Berlin berufen und sich von da an - dank seiner versierten Rollenpluralität als Lyriker, Akademiker, Zeitschriftgründer bzw. -herausgeber - als einer der wichtigsten Akteure des Berliner Literaturlebens behaupten, der das kulturelle Klima der Stadt sowie der BRD entscheidend und langfristig mitprägen sollte.

Bachmann ist in diesem Rahmen eine wichtige wahlverwandte Komplizin der ersten Stunde. Noch vor der Einführung des Förderprogramms Artists in Residence der Ford-Stiftung lädt Höllerer sie nach Berlin zu zwei der programmatischen Veranstaltungen ein, mit denen er auf eine grundsätzliche Transformation der Literaturpraxis abzielt.

Die erste Edition der Lesereihe Literatur im technischen Zeitalter, an die er die Gründung sowohl der Zeitschrift Sprache im technischen Zeitalter als auch des gleichnamigen Instituts anknüpft, findet im Januar 1960 als offenes Event in den Gebäuden der Technischen Universität statt und wird zu einem sensationellen Erfolg, zu dem die Präsenz von Bachmann als Ko-Protagonistin des Eröffnungsabends zusammen mit

7 Vgl. Klaus Wagner: Stenogramm der Zeit. Gedichte aus dem deutschen Ghetto, in: Der Spiegel 34 (August 1954), 26-29, hier: 28. Vgl. dazu u. a. Ulrike Draesner: Möblierte Mädchen, in: Reinhard Baumgart, Thomas Tebbe (Hrsg.): Einsam sind alle Brücken. Autoren schreiben über Ingeborg Bachmann, München 2001, 124-137; Wilhelm Hemecker, Manfred Mittermayer (Hrsg.): Mythos Bachmann. Zwischen Inszenierung und Selbstinszenierung, Wien 2011.

8 Helmut Böttiger: Elefantenrunden. Walter Höllerer und die Erfindung des Literaturbetriebs. Unter Mitarbeit von Lutz Dittrich. Berlin 2005, 57. Siehe auch: Achim Geisenhanslüke, Michael Peter Hehl: Poetik im technischen Zeitalter. Walter Höllerer und die Entstehung des Literaturbetriebs, Bielefeld 2013. 
dem Schweizer Schriftsteller Max Frisch aktiv beiträgt. ${ }^{9}$ Ähnliches gilt für die zweite Edition, die ab November 1961 zu einer internationalen Erweiterung des Konzepts führt und diesmal durch einen Soloauftritt von Bachmann in der Kongresshalle inauguriert wird - aufgrund ihrer offiziellen Staatsangehörigkeit gilt sie in diesem Rahmen als Stimme aus dem Ausland, so wissen mittlerweile auch die Journalist:innen sie mit differenzierender Apostrophe als ,jugendliche First Lady der deutschsprachigen Dichtung “ $\mathrm{zu}$ feiern. ${ }^{10}$

Betrachtet Höllerer sein experimentelles Programm als Chance „einiges neu zu sehen, einiges zu verändern - “11, so bleiben die Formel der direkten Interaktion mit Autor:innen sowie der Anspruch auf eine Erweiterung des sozialen Wirkungsradius der Künste bestimmende Qualitäten seiner kulturellen Regie, die auch in der parallelen Gründung des Literarischen Colloquiums Berlin ihre wirksame Anwendung finden. Im Dialog mit dem „Mann mit den Millionen“12 alias Shepard Stone, Direktor der Abteilung für internationale Angelegenheiten der Ford Foundation, weiß Höllerer die amerikanischen Geldquellen für die eigenen Pläne fruchtbar zu machen - er ist vermutlich auch mehr oder minder direkt beteiligt an der Auswahl von wenigstens einigen der Kandidat:innen des Programms Artists in Residence. ${ }^{13}$

Während sein optimistischer Unternehmungsgeist auf den kulturellen Nachholbedarf der Westberliner setzt, sorgt allerdings insbesondere die düstere Stimmung der ersten Mauerjahre für ein eher negatives Feedback seitens der meisten Neuankömmlinge. ${ }^{14}$ Bachmanns Fall als erste Stipendiatin der Reihe ist jedoch anders: Zugleich „dankbar und undankbar“ wegen des „kleinen Geldregens“ (KS, 481), ist sie sich von Beginn an ihrer besonderen Situation bewusst, spielt doch bei ihr die österreichische Provenienz im Sinne der belastenden Erinnerung an die verwandte Geschichte eine maßgebliche Rolle.

Bereits bei ihrem ersten Aufenthalt im Herbst 1961 war nicht von ungefähr unter dem Stichwort Sterben für Berlin die bedrückende Atmosphäre der Stadt zum Inspirationsstoff eines unvollendeten Prosaentwurfs geworden. Verweist der Arbeitstitel der Erzählung auf die Aktualität eines Spiegel-Artikels, in dem die

9 Böttiger (Anm. 8), $125 \mathrm{ff}$.

10 Ebd., 128.

11 Ebd., 132.

12 Ebd., 168.

13 Inwiefern Höllerer bei der Einladung Bachmanns mitgewirkt haben mag, ist nicht ganz eindeutig zu eruieren. Zur transatlantischen Kulturpolitik der USA mit Schwerpunkt Deutschland vgl. u. a. Jan Bürger: The Kissinger Boys. Von der Harvard Summer School zur Suhrkamp Culture. In: Zeitschrift für Ideengeschichte XI/4 (2017), 5-18. Siehe auch: Ingeborg Bachmann, Hans Magnus Enzensberger: „schreib alles was wahr ist auf“. Der Briefwechsel, hrsg. von Hubert Lengauer, München/Berlin/Zürich 2018, 294.

14 Vgl. Cornelia Jacobsen: „Halbzeit bei der Ford-Stiftung. Warum viele der eingeladenen Künstler in Berlin unzufrieden sind“, in: Die Zeit 40 (02.10.1964). 
„Wahrscheinlichkeit eines Heißen Krieges“ aus der Sicht der amerikanischen Soldaten „für den Ernstfall“15 geschildert wird, so transformiert Bachmann hier die militärische Valenz des Todesbildes in eine Anspielung auf das psychosomatische Syndrom ihres Protagonisten, eines Fremden auf Berufsreise, der direkt bei der Landung am Flughafen Tempelhof „Anzeichen von Verwüstung, von Unglücklichsein“ (TKA I, 77) spürt. Die gerade erst gebaute Mauer wirkt sich als subkutane Verhaltensstörung aus, so dass der Mann nicht einmal in der Lage ist, aus dem Fenster zu schauen und überhaupt etwas zu sehen: „c'est toujours la brume“ - einziger Ausweg, der ihn mangels praktikabler Alternativen als utopische Rettungsphantasie zu trösten scheint: „eine Megatonne Zärtlichkeit über der Stadt auszulösen“. (TKA I, 75)

\section{„Europäisch denken?“: Konzeption und Programmatik einer „non-revue“}

Als Bachmann im April 1963 zusammen mit dem Schriftstellerkollegen Uwe Johnson und dem Suhrkamp-Lektor Walter Boehlich nach Paris fährt, ist das europäische Projekt der Revue internationale bereits in eine kritische Phase geraten. Die spielerische Inversion „non-revue“16 stammt in diesem Zusammenhang vom italienischen Schriftsteller Francesco Leonetti, einem der drei Mitglieder des trilateralen Redaktionskollegiums, als kritische Bilanzierung der sich lange hinziehenden Phase der Entwürfe und programmatischen Thesen, die sich ab einem bestimmten Punkt in einer selbstreferentiell unproduktiven Dynamik - Denken anstatt Handeln - verfahren haben, wodurch das konkrete Ziel der Zeitschriftengründung verfehlt worden sei. ${ }^{17}$

Etliche Archivdokumente, die im Lauf der letzten zwei Jahrzehnte erschlossen wurden, gewähren inzwischen Einblick in die Stationen dieser noch relativ wenig erforschten Unternehmung und liefern eine interessante Vorlage für eine retrospektive Anerkennung ihres pionierhaften transnationalen Charakters. ${ }^{18}$

15 Stewart Alsop: „Sterben für Berlin. Die Frontstadt-Planer des Weißen Hauses“, in: Der Spiegel 47 (1961), 73-79, hier: 73.

16 KS, 388 und Francesco Leonetti: Brief an Elio Vittorini vom 12.11.1963, zit. nach: Maria Temperini (Hrsg.): Gulliver. Carte Vittorini in Europa nel Sessanta (1961-1967), Milano/Lecce 2000, 127-130, hier: 130; sowie ders.: Una rivista internazionale. Osservazioni, in: Gulliver / il menabò (Anm. 3), IX-XVI. 17 Ebd.

18 Neben den Primärquellen, (die unter dem Titel Dossier de la Revue internationale in: Lignes 3 (1990), hrsg. von Michel Surya, 159-301, zu lesen sind), siehe insbesondere: Anna Panicali (Hrsg.): Gulliver. Progetto di una rivista internazionale, Milano 2003; Henning Marmulla: Internationalisierung der Intellektuellen? Möglichkeiten und Grenzen einer „commonauté internationale“ nach dem Algerienkrieg, in: Ingrid Gilcher-Holtey (Hrsg.): Zwischen den Fronten. Positionskämpfe europäischer Intellektueller im 20. Jahrhundert, Berlin 2006, 179-200; Roman Schmidt: Die unmögliche 
Faszinierend wirkt bis heute nicht nur das eklektische Gruppenbild der illustren Protagonist:innen, ${ }^{19}$ sondern auch der ursprünglich durchaus konstruktive, undogmatische Elan aller Beteiligten: eine allgemeine Unzufriedenheit mit dem Status Quo, ein marxistisch geschulter Blick auf die globalen Auswirkungen der Dekolonialisierung und Entstalinisierung im Spannungsfeld des Kalten Kriegs, ein intuitives Bewusstsein über Ansprüche und Widersprüche ihres literaturkritischen Vorhabens und dennoch eine kollektive Bereitschaft, intellektuelle Energien in die gemeinsame Sache zu investieren - Maurice Blanchot, dem sich der erste programmatische Schritt verdankt, hatte von vornherein den intrinsischen Fluchtpunkt des Wagnisses signalisiert: „et, ci c'est une utopie, accepter d'échouer utopiquement“.20

Als Initialzündung gilt 1960 die Solidaritätsaktion um das sogenannte Manifest der 121 beziehungsweise die Déclaration sur le droit à l'insoumission dans la guerre d'Algerie, welche als politische Unterstützung des algerischen Volks in seinem Unabhängigkeitskrieg gegen Frankreich eine breite Resonanz auf europäischem Boden auslöst. Es ist der Geist dieses kollektiven Aktionismus, in dem Blanchot das entsprechend günstige Terrain für die Gründung eines neuen Organons jenseits der konsolidierten Formel der littérature engagé erblickt. Was ihm als Erbe der surrealistischen Avantgarde und Anhänger der romantischen Tradition vorschwebt, ist die experimentelle Suche nach neuen Ausdrucks- und Kommunikationsmodalitäten über die Nationalgrenzen hinaus, wobei zwei komplementäre Prioritäten im Mittelpunkt seines Programms stehen - zum einen die Betrachtung der Aktualität („le cours des choses“, „die Chronik der Zeit“);

Gemeinschaft. Maurice Blanchot, die Gruppe der rue Saint-Benoît und die Idee einer internationalen Zeitschrift, Berlin 2009; Silvia Cavalli: An International „Non-revue“. Cultural Conflict and the Failure of Gulliver (1964), in: Journal of European Periodical Studies 3/1 (2018), 67-80; Christian Luckscheiter: Das transnationale Zeitschriftenprojekt Gulliver - und sein Scheitern an nationalen Akzenten, in: Michael Peter Hehl, Heribert Tommek (Hrsg.): Transnationale Akzente. Zur vermittelnden Funktion von Literatur- und Kulturzeitschriften im Europa des 20. Jahrhunderts, Berlin u. a. 2021.

19 Zur Redaktion zählen 1963 nach Angabe von Leonetti (in alphabetischer Ordnung) Robert Antelme, Ingeborg Bachmann, Maurice Blanchot, Michel Butor, Italo Calvino, Louis-René des Forêts, Hans Magnus Enzensberger, Günter Grass, Helmut Heißenbüttel, Uwe Johnson, Michel Leiris, Francesco Leonetti, Dionys Mascolo, Alberto Moravia, Maurice Nadeau, Pier Paolo Pasolini, Geneviève Serreau, Elio Vittorini, Martin Walser. Vgl. Francesco Leonetti: Gulliver, una rivista internazionale non compiuta nell'inizio degli anni '60, in: Che fare 8/9 (1971), 327-329. Siehe auch: Schmidt (Anm. 18), 75, der insgesamt 22 Autorennamen auflistet. An der programmatischen Phase beteiligt sich auch der polnische Philosoph Leszek Kolakowski. Der Plan Blanchots ist allerdings von vornherein, weitere Mitarbeiter:innen sowohl aus den osteuropäischen Ländern als auch aus dem anglo-amerikanischen und spanischsprechenden Bereich zu gewinnen. Vgl. Maurice Blanchot: Textes préparatoires, lignes, définitions de la Revue internationale in: Lignes (Anm. 18), 179-191, hier: 186.

20 ,und falls es sich um eine Utopie handelt, müssen wir hinnehmen, auf utopische Weise zu scheitern“. (Übersetzung von V.D.R.) Vgl. Blanchot, Textes préparatoires, in: Lignes (Anm. 18), 179-191, hier: 180. 
zum anderen die literarische Praxis („l'écriture, „die Schreibweise“) in ihrer doppelten Funktion als sprachästhetisches und sprachkritisches Verfahren. ${ }^{21}$

Die Präsenz von Autoren wie Michel Butor, Dyonis Mascolo und Robert Antelme an Blanchots Seite zeugt von der Nachbarschaft seiner ideologischen Orientierung einerseits mit den antizipierend dekonstruktiven Tendenzen des nouveau roman, andererseits mit dem heterodoxen Spektrum der antifaschistischen Militanz im Zweiten Weltkrieg, und im Besonderen mit der sogenannten Gruppe der Rue de Saint Benoît, (die Pariser Adresse von Marguerite Duras), die sich in den selben Jahren als Synonym für eine künstlerische Enklave am Rande der kommunistischen Parteizugehörigkeit konsolidiert hatte. ${ }^{22}$

Eine der ersten Brücken wird dank der persönlichen Freundschaft zwischen Blanchot und Elio Vittorini nach Italien geschlagen. Als Hauptrepräsentant der italienischen Gruppe kommt Vittorini selber aus der Erfahrung des Widerstands gegen den Nazifaschismus und spielt in der kulturellen Landschaft in den 1950er und frühen 1960er Jahre nicht nur dank diesem symbolischen Kapital eine entscheidende Rolle, sowohl als Autor einer innovativen Literatur jenseits des vorherrschenden neorealistischen Kanons wie auch als einflussreicher Verlagsredakteur und Zeitschriftenherausgeber.

Sein Memorandum zur Realisierung der Revue erweist eine bedeutsame Kontinuität mit dem Programm, das er zusammen mit Italo Calvino als Mitherausgeber der Zeitschrift il menabò (1959-1967) erarbeitet hatte, und sorgt im Dialog mit den französischen und deutschsprachigen Kolleg:innen für eine dezidierte Akzentuierung der sozialkritischen Aspekte des gemeinsamen Vorhabens. Sein Schwerpunkt liegt dabei auf der angestrebten Neubestimmung von Funktion und Rolle des Intellektuellen in den neokapitalistischen Gesellschaften der Nachkriegszeit. Angesichts der tiefgreifenden, durch das Wirtschaftswunder bedingten sozio-anthropologischen Transformationen erweist sich das Medium Literatur in seinen Augen als einzig kreativer Gegenentwurf zur Standardisierung der Sprache, die mit der durch den Wohlstand bedingten Standardisierung der Denk- und Lebensmodelle einhergehe: „Par rapport aux divers langages culturels, du langage politique au langage mathématique, le langage littéraire a du reste l'avantage d'être le seul global. Tous les autres langages culturels sont séparatises. Le littéraire seul est unitaire“. ${ }^{23}$

In einer zunächst durchaus kompatiblen Nähe zu Blanchot und Vittorini steht auch Enzensbergers Anspruch auf eine Neudefinition der Kulturkritik im Rahmen des Strukturwandels der damit verknüpften Medien- und Konsumkultur. Sein Plädoyer

21 Ebd.

$22 \mathrm{Vgl}$. im besonderen Schmidt (Anm. 18), $40 \mathrm{ff}$.

23 „Im Vergleich zu anderen Sprachmedien - wie jene der Politik oder der Mathematik - hat das literarische Sprachmedium als einziges den Vorteil, global zu sein. Alle anderen kulturellen Sprachmedien sind sezessionistisch. Das literarische ist das einzige einheitliche Sprachmedium“. (Übersetzung von V.D.R.) Vgl. Elio Vittorini: Contribution à un projet de préface pur une revue internationale, in: Lignes (Anm. 18), 204-208, hier: 207-208. 
für das agile Kommunikationsformat der „recension“ liest sich in direkter Relation mit den parallel erarbeiteten Thesen zu den vorherrschenden Mechanismen der Bewusstseinsindustrie ${ }^{24}$ :

es besteht kein grund, die politik den leitartikeln, die reportage den reportern, jedes gebiet den zuständigen fachleuten zu überlassen ... fiction und non-fiction sollen ebenbürtig nebeneinandertreten, ein neues gedicht neben die analyse eines neuen automodells, ein essay über arzneimittelwerbung neben ein hörspiel ... wie gemeinhin nur romane, so sollen hier häuser, sechtstagerennen, supermärkte, industriekonzerne, käsesorten, professoren, fernsehstars, kurz es soll realität rezensiert werden. ${ }^{25}$

Bestimmend für die Konnotation der spezifischen Identitätsmerkmale der deutschen Gruppe, in deren Namen Enzensberger spricht, ist dabei das Generationenporträt, das er durch einen lakonischen Verweis auf den gemeinsamen ästhetischen und zugleich politischen Erfahrungshintergrund einprägsam skizziert: die langfristige Hypothek der deutschen Geschichte, die befürchtete Isolation in einer provinziellen Enge und - als symbolisches Memento - die spektrale Topographie der ehemaligen Hauptstadt mit dem Niemandsland ihrer leeren Mitte: „la Place de Potsdam““.

So markiert spätestens ab August 1961 das Ereignis des Mauerbaus als akute Dramatisierung der europäischen Krisenlage eine Wende in der internen Diskussion des trilateralen Redaktionskomitees. Im Sinne der anvisierten ,Chronik der Zeit‘ avanciert die Stadt Berlin nicht nur in den Augen Blanchots zum konkreten Ort des historischen Präsens, in dem sachliche und metaphorische Implikationen, Diskurs und Metadiskurs faktisch in eins zu fallen scheinen.

Inwieweit jedoch die Fragmentierung der Welt zu einer konsequenten Fragmentierung der Schreibweise führen soll, wird relativ schnell zur kontroversen Materie von ideologischen Auseinandersetzungen, in denen insbesondere die Divergenzen zwischen der deutschen und der französischen Gruppe zum Ausdruck kommen. ${ }^{27}$

24 Hans Magnus Enzensberger: Gulliver (1961-1963), in: ders.: Meine Lieblings-Flops, gefolgt von einem Ideen-Magazin, Berlin 2011, 120-127, hier: 120. Hier zitiert Enzensberger das deutsche Originaldokument aus seinem Archiv. (Vgl. ders.: Possibilité et necessité d’une nouvelle revue, in: Lignes [Anm. 18], 192-195, sowie ders: Einzelheiten I. Bewußteins-Industrie, Frankfurt a. M. 1962.)

25 Enzensberger, Gulliver (Anm. 24), 124-126.

26 Enzensberger, Possibilité et necessité, in: Lignes (Anm. 18), 192.

27 Die intensivste Vorbereitungsphase liegt zwischen Ende 1961 und Ende 1962. Ab 1962 leisten die drei Autoren Francesco Leonetti, Uwe Johnson, Louis René de Forêst im Hinblick auf die Publikation der ersten Nummer die gesamte Koordinationsarbeit. So kündigen im Sommer 1962 sowohl The Observer in England als auch Der Spiegel in Deutschland die baldige Erscheinung einer neuen EuropaZeitschrift an, und zwar als gemeinsame Initiative von drei Verlagen: in Deutschland Suhrkamp, in Frankreich Gallimard, in Italien Einaudi, die jeweils drei verschiedene Editionen in den drei Sprachen garantieren sollen. Nach einer eher schwierigen Verhandlungsphase, in der sich die Positionen definitiv auseinander zu entwickeln scheinen - nicht zuletzt wegen des Rücktritts von Gallimard, der durch den Verlag Julliard ersetzt wird - bahnt sich mit dem Jahreswechsel 1962/1963 eine neue kons- 
Davon, dass tendenziell grundverschiedene, mitunter gar unversöhnliche Ideenund Stiltraditionen unter dem selben Dach zu koexistieren versuchen, zeugt der geradezu diffizile Gestaltungsprozess der ersten Nummer - und dies nicht nur im Hinblick auf die zusammenzustellenden Inhalte, sondern auch mit Bezug auf die Titelsuche; so soll nach Meinung der trilateralen Gruppe erst recht der Zeitschriftenname eine „transnationale“ semantische Wirkung zu erzeugen wissen. ${ }^{28}$

Während die französischen Vorschläge (darunter ,Discourses‘, ,L'autre revue‘) auf die Priorität des methodischen Ansatzes anspielen, kommen als Alternativoptionen von deutscher Seite eher ,allgemein bedeutende“ Ortsnamen (darunter ,Potsdamer Platz‘, ,Provinz‘, ,Guernica‘), die zwischen den Zeilen den Fokus auf die Implikationen der deutschen Gegenwart und Geschichte umlenken. Die Italiener, die sich in dem sich allmählich zuspitzenden deutsch-französischen Disput in einer Zwischenposition sehen, neigen eher zu einer schlichten Zahlenangabe („I 60“), die sich sowohl als Definition des laufenden Jahrzehnts, als auch als Rückbesinnung auf das lateinische sexaginta - im übertragenen Sinn ein Wort für ,Pluralität ${ }^{\star}$ - deuten lassen könne. Zum Schluss einigt man sich, wenn auch nicht mit einheitlichem Enthusiasmus - Bachmann etwa findet die Lösung „fatal“ -, auf die durch Günter Grass suggerierte Chiffre Gulliver, welche die sachliche Disproportion ,zwischen intellektueller Arbeit und bürgerlicher Macht“ (Leonetti) veranschaulichen soll. ${ }^{29}$

\footnotetext{
truktive Phase an. Es ist in diesem Zusammenhang, dass die zwei Treffen - das erste im Januar 1963 in Zürich, das zweite im April 1963 in Paris - geplant werden. Vgl. hierzu insbesondere Anna Panicali: Cronaca attraverso le lettere (1960-1966), in dies.: Gulliver (Anm. 18), 17-55 sowie Uwe Johnson, Siegfried Unseld: Der Briefwechsel, hrsg. von Eberhard Fahlke und Raimund Fellinger, Frankfurt a. M. 1199, 1094-1136.

28 Zur terminologischen Differenz zwischen den beiden Kategorien „international“/,transnational“ vgl. insbesondere Schmidt (Anm. 18), 72 ff. Als „Verhandlungssprache“ der Redaktionsgruppe gilt Französisch, insofern die programmatischen Thesen zur Revue auf Französisch zirkulieren. Uwe Johnson verwendet gelegentlich (vermutlich als deutsche Übertragung des französischen Adjektivs „supernational“) den Terminus ,übernational“. Vgl. Johnson, Unseld (Anm. 27), 1127.

29 Vgl. Eberhard Fahlke: Ein namenloses Projekt mit vielen Namen, in ders: „Un vocabolo tedesco“. Uwe Johnsons deutscher Beitrag zum Projekt einer europäischen Zeitschrift, in: Sprache im technischen Zeitalter 114 (1990), 108-115, hier: 108-111; Francesco Leonetti: Una rivista internazionale, in: Gulliver/il menabò, [Anm. 4], XVI und ders.: Appunti di lavoro di Vittorini nella preparazione redazionale della rivista Gulliver (1961-1963), in: Il Ponte XXIX/ 7-8 (1973), 1172-1178, hier: 1174; Alexander Karasek: Mein Gulliver. Zu einem Gedichtentwurf von Günter Grass, in: Sinn und Form 3 (2007), 393-397. Grass selbst verfasst ein einschlägiges programmatisches Gedicht Mein Gulliver, in dem die satirische Ader Swifts ihm dazu dient, durch eine paradoxe Rollen- und Sinnumkehr dem „Zwergenstolz“ Ausdruck zu verleihen und dabei am Beispiel des Wortes „Schustertunke“ die prinzipielle Unübersetzbarkeit der Dialekte zu versinnbildlichen (ebd., 394-395). In der Tat, wie Karasek anmerkt, fehlt in der italienischen Übertragung des Gedichts die entsprechende Strophe - „Lost in translation“ (ebd. 395; vgl. Leonetti: Una rivista internazionale, in: Gulliver/il menabò [Anm. 3], XVI)].
} 
In der Redaktionskonferenz von Paris meint Johnson, entsprechend der zunehmenden Uneinigkeiten eine generelle „Glaubensschwäche hinsichtlich der Lebensfähigkeit“ des Projekts zu registrieren. ${ }^{30}$

Zur Debatte steht die Auswahl der Beiträge für das erste Gulliver-Heft, mit einem besonderen Bezugnahme auf die „Fragmentideologie“ Blanchots sowie der anderen französischen Texte, denen zumal nach der Lesart der deutschen Gruppe ein spekulativer und insofern allzu elitärer Hermetismus vorgeworfen wird. Entlang solch konfliktueller Argumente entfaltet sich eine zwei Tage lange Diskussion, die sowohl im minutiösen Protokoll Johnsons ihren Niederschlag findet, (,Viel Wortwechsel wurde hervorgerufen durch Missverständnis der Worte INDIREKT, ABSTRAKTION [...].“), ${ }^{31}$ als auch in den knappen Notizen Bachmanns: „Sehr unglücklich, daß derartige Diskussion möglich. Indirekt, direkt, abstrakt, konkret. Ebenso gegenseitige Versicherungen, daß man Zeit investiert und Mühe, unter Erwachsenen nicht anders möglich. Tragischer échec“. (KS, 397) ${ }^{32}$

Die endgültige Montage des Hefts, das von Vittorini und Calvino in der von ihnen herausgegebenen Zeitschrift il menabò als „Känguru-Nummer“33 aufgenommen wird, bietet eine anthologische Synthese der zeit- und sprachkritischen Philosophie/n der trilateralen Gruppe, welche in dieser geradezu einmaligen Publikationsform ein sehr interessantes Kapitel der Kulturgeschichte der frühen 1960er Jahre darstellen.

Das Spektrum der Themenkomplexe und Fragehorizonte erweist sich als äußerst disparat, allerdings deswegen nicht weniger denkwürdig: So reicht die Spannbreite (um nur ein paar Beispiele zu erwähnen) von der sowjetischen Eroberung des Weltalls (Maurice Blanchot) bis zur Urbanisierung der ruralen Gesellschaften Italiens (Giorgio Bocca), von der Stellung Oedipus' zwischen Sophokles und Freud (Roland Barthes) bis zu den dialektalen Verhältnissen in den Vororten Roms (Pier Paolo Pasolini), von der Dialektik des Verrats im Kontext von Politik und Verbrechen (Hans Magnus Enzensberger) bis zum ästhetischen Wert der Ruinen (Jean Starobinski), von den Literaturprogrammen im Fernsehen (Louis-René des Forêts) bis zur lähmenden Wirkung des Wohlstands auf den sozialen Antagonismus (Italo Calvino).

30 Spätestens ab Dezember 1962 macht Johnson in seinen Berichten kein Hehl aus seinem Misstrauen gegenüber den französischen Kollegen. Vgl. Johnson, Unseld (Anm. 27), 1114 ff.

31 Ebd., 1123-1135, hier: 1125.

32 Es ist dies auch der Punkt, an dem Enzensberger aussteigt. Das Projekt rubriziert er in seiner Autobiographie unter dem Stichwort „versuch einer antizipation à presque tout prix“, das im Sinne eines produktiven Frusts als wichtige Zwischenstation auf dem Weg zur Gründung der eigenen Zeitschrift Kursbuch hervorgehoben wird. Vgl. Hans Magnus Enzensberger: Gulliver (Anm. 24), 120-127, hier: 125. Die Kooperation mit den italienischen Kolleg:innen wird er allerdings nicht aufgeben: 1966 wird er unter dem Titel Letteratura come storiografia [Literatur als Geschichtsschreibung] eine monographische Nummer der Zeitschrift il menabò in der Rolle des Gastredakteurs herausgeben und darin eine Anthologie der neuesten deutschen Literatur vorstellen.

33 Elio Vittorini: Letteratura arte società. Articoli e interventi 1938-1965, hrsg. von Raffaella Rodondi, Torino 2008, 1034-1036, hier: 1035. 
Eine zentrale Sektion des Hefts bildet darüber hinaus der neuralgische BerlinKomplex, dem zwei Beiträge - von Maurice Blanchot und Uwe Johnson - gewidmet sind, die trotz der ideologischen Inkompatibilitäten der beiden Autoren eine indirekte Analogie aufweisen, insofern sie sich beide mit dem literaturästhetischen Konsequenzen der Mauer als materielle und symbolische Zäsur beschäftigen. Sieht Blanchot in der Teilung der Stadt ein „metaphysisches Problem“, das den „Namen Berlin“ in eine Art sprachliche Leerstelle verwandelt habe, so kreist Johnsons Text um die „sprachliche Spaltung“, die sich durch die Anomalie der deutschen Zweistaatlichkeit ergeben habe und die durch die befremdliche Aporie des politisch-administrativen Terminus „GESAMTDEUTSCH“ nicht vorzutäuschen sei. ${ }^{34}$

Eingerahmt sind die Beiträge des Gulliver-Hefts durch ein Vorwort von Vittorini und ein Nachwort von Leonetti, die als Rekapitulation und Bilanzierung der Kurzgeschichte der „non-revue“ gedacht sind, wobei beide redaktionelle Kommentare nicht so sehr das Scheitern betonen, als vielmehr die nicht erschöpften Potentialitäten des ursprünglichen Vorhabens.

Vittorinis Worten zufolge besteht der größte Verdienst der Unternehmung als „Metazeitschrift der Zukunft“ in der offenen Risikobereitschaft abseits der „beruhigenden Gewissheit der Affinitäten“, während Leonettis Lektüre trotz der Konstatierung kulturgeschichtlicher Differenzen auf die Existenz von Elementen einer „intersubjektiven Übereinstimmung“ innerhalb der trilateralen Autorenkonstellation hinweist - und zwar ungeachtet der jeweiligen nationalen Zugehörigkeiten. ${ }^{35}$

Nicht nur weiß eine solch (selbst-)kritisch-konstruktive Perspektivierung dem ursprünglich genuin transnationalen Elan des Projekts Rechnung zu tragen, sie macht auch - über die Bilanz des Scheiterns hinaus - den Blick frei für die Erkundung textueller Verwandtschaften jenseits der zwischenpersönlichen Konflikte und Idiosynskrasien. In Bachmanns Fall als österreichische Dichterin in ihrer ohnehin ,transversalen' Position ermöglicht dieser Ansatz, bedeutsame Berührungspunkte ihrer Gulliver-Reflexionen sowohl mit Blanchots Utopismus - nicht von ungefähr sind sie beide Bewunderer des Dichters René Char $^{36}$ - als auch mit einigen der literatur- und sprachkritischen Thesen der italienischen Gesprächspartner zuerkennen.

34 In seinem Beitrag nimmt Blanchot expliziten Bezug auf Johnsons Romane als Beispiele einer Annäherung an die „Einzigartigkeit“ von Berlin. Vgl. Maurice Blanchot: Il nome Berlino, in: Gulliver/ il menabò (Anm. 3), 121-125; für die französische Originalfassung, (die lange als verschollen galt), vgl. ders.: Le nom de Berlin, in: Lignes 3/3 (2003), 129-141; Uwe Johnson: Un vocabolo tedesco, in: Gulliver/il menabò (Anm. 3), 111-121.; für die deutsche Originalfassung vgl. ders.: Gesamtdeutsch, provinziell, in: Sprache im technischen Zeitalter 114 (1990), 116-122; sowie Eberhard Fahlke: „Un vocabolo tedesco“. Uwe Johnsons deutscher Beitrag zum Projekt einer europäischen Zeitschrift, ebd., 108-115. 35 Elio Vittorini: [editorische Notiz ohne Seitenangaben], in: Gulliver/il menabò (Anm. 3); Francesco Leonetti: ebd., XXVI.

36 Die Frankfurter Vorlesungen münden bekanntlich in der programmatischen Zitatparaphrase aus Chars: „Auf den Zusammenbruch aller Beweise antwortet der Dichter mit einer Salve Zukunft“. 


\section{Zwischen „Dialekt und Dialektik“: Zeitkritik als Sprachkritik}

In der italienischen Fassung verweist der Titel von Bachmanns eigenem GulliverBeitrag auf eine intertextuelle Adaption: Diario in pubblico ist wörtliches Zitat der 1957 erschienenen Essaysammlung von Vittorini, die sich im Zeichen einer programmatischen Diskontinuität als frei assoziierende Gratwanderung zwischen privaten und öffentlichen Angelegenheiten, objektivierenden Analysen und subjektiven Akzentsetzungen charakterisiert. ${ }^{37}$

Im Hinblick auf den Entstehungszusammenhang des Texts repräsentiert der Assoziationstransfer von Vittorinis Vorlage eine kongeniale Nebenspur hinsichtlich des offenen Umgangs mit den formalen Konventionen des Tagebuchs als literarischer Gattung, die Bachmanns Geste durchaus entspricht, verzichtet sie hier in der Rekonstruktion des kollektiven Zeitschriftenprojekts auf die Prärogativen des Ichs als erste Person. „Europäischsein“, „,sich europäisch fühlen“ und „europäisch denken“ gelten hierbei als leitmotivische Stichworte, die leicht variiert - mal mit einem problematisierenden Fragezeichen versehen, mal mit affirmativem Gestus behauptet - den ganzen Aufsatz durchziehen, und zwar insbesondere da, wo es Bachmann darum geht, die grundlegenden Fundamente ihrer Ästhetik nachdrücklich zu betonen: „Denken, gewiß, auch historisch denken und vor allem utopisch denken“. (KS, 388) ${ }^{38}$

Inhaltlich fungiert indes der Rückgriff auf Brechts Chiffre des Radwechsels als poetologischer Kompass im Sinne eines selbstreflexiven Gestus, der mit einer kritischen Selbstverortung in der historisch-politischen Situation der Gegenwart einhergeht. Was Brechts ,Chronik der Zeit‘ anbelangt, so inszeniert er die Position seines lyrischen Ichs in einem nur scheinbar statischen Abseits, wobei der lakonisch gehaltene Ton der symmetrischen Aussagen (,Ich bin nicht gern, wo ich herkomme / ich bin nicht gern, wo ich hinfahre“) eine eigentümliche Spannung - zwischen Denken und Handeln, Vergangenheit und Gegenwart, Herkunft und Ziel - zu erzeugen vermag, während das offene Fragezeichen für eine produktive Öffnung des Finalen sorgt: „Warum sehe ich den Radwechsel / mit Ungeduld?“39

(KS, 349) Seinerseits rezensiert Blanchot zwei Gedichtsammlungen René Chars (Poème pulvérisé und La parole en archipel) für das Gulliver-Heft. Vgl. Maurice Blanchot: La parola in arcipelago, in: Gulliver/il menabò (Anm. 3) 156-159.

37 Auf Vittorinis Diario in pubblico als Modell einer „neuen Literatur“, das sich dank seiner formalen Diskontinuität als instruktiver Referenzpunkt für das literaturästhetische Programm der Zeitschrift eigne, hatte bereits Leonetti verwiesen. Zit. nach: Anna Panicali, Cronaca attraverso le lettere, in Gulliver (Anm. 18), 17-55, hier: 31.

38 Hier (KS, 388) auch die Wendung „Dialekt und Dialektik“ aus der Zwischenüberschrift.

39 Bertolt Brecht: Buckower Elegien. Mit Kommentaren von Jan Knopf, Frankfurt a. M. 1986, 9. Ein utopischer Wesenszug sei Brechts Lyrik eigen: In diesem Sinn charakterisiert Bachmann dessen Werk 
In der Logik des Bachmann'schen Essays eignet sich diese Situation des Radwechsels - vor allem der Akzent auf die latente Energie der Ungeduld - dazu, den Engpass des Kalten Kriegs zwanzig Jahre nach Ende des Zweiten Weltkriegs zu veranschaulichen, um die eigene kritisch-konstruktive Vorstellung von Transnationalität als Kategorie des Friedens darin zu verankern. Negation und Affirmation, Zeitkritik und Sprachkritik gehen hierbei Hand in Hand.

Hinsichtlich der pars destruens ihrer Argumente macht Bachmann die Kritik der bestehenden Nachkriegsordnung an den mystifizierenden Parolen der Propagandadiskurse nach 1945 fest: Davon speise sich ein falsches Selbstverständnis von Europa beziehungsweise des geteilten Europas, das hier durch Verweis auf ein entsprechendes Repertoire von leeren,Worten und Werten' Schritt für Schritt systematisch dekonstruiert wird.

Einerseits handelte es sich um selbstgefällige Attitüden der humanistischen Tradition des Bildungsbürgertums (,„Weltoffenheit““, „museale Ergriffenheit“ „frohe[r] Fortschrittsglauben“ KS, 387), die stigmatisiert werden, insofern sie den Weg in die Barbarei nicht haben verhindern können, andererseits um instrumentalisierte Begriffe der politisch-ökonomischen Agenda („Verbrüderungsgefühle“, „Versöhnung“, „Verständigung“ KS, 384), die im Zeichen eines rein „pragmatische[n] Flachsinn[s]“ (KS 393) lediglich zur Konstruktion und Erhaltung eines (hier höhnisch diskreditierten) „Supermarkt[es] Europa“ (KS, 387) dienen, als wäre überhaupt Europa „zu einem Gegenstand geworden, dem sich nur mehr Experten nähern dürften“ (KS, 392). ${ }^{40}$

Bleibt Bachmanns Blick hauptsächlich auf die „Krankheit Westeuropas“ (KS, 393) konzentriert, so umfasst ihre kritische Perspektive zugleich die gesamte Lage des gespaltenen Kontinents mitten in der nicht nur ideologischen Konfrontation des Kalten Kriegs: ein „Vorposten Teileuropa gegen einen Vorposten Teileuropa, von dem sich der eine dem anderen scharfsinnig plausibel machen muß als geharnischter Vertreter von Frieden und Freiheit“. (KS, 394)

Die pars construens involviert als Gegenentwurf die Vorstellung einer transnationalen Gemeinschaft, die Bachmann mit einem konkreten Bezug auf die trilaterale

als „katastrophische[n], zerrissene[n], grandiose[n] Rettungsversuch“ (KS, 460) und den Dichter selbst als Verbündeten im „tausendjährigen Versuch gegen die schlechte Sprache“. „Der Radwechsel“ sollte auch der ursprüngliche Titel ihres Essays sein. Vgl. dazu auch Enzensbergers Bemerkungen zum Text, den er wegen exzessiver Länge und mangelnder Stringenz „brauchbar aber nicht ideal“ findet. Vgl. Bachmann/Enzensberger (Anm. 13), S. 295.

40 Die als „Entwürfe zur politischen Sprachkritik“ rubrizierten Nachlassnotizen erweisen in manchen Punkten direkte Verknüpfungen mit den „Tagebuchs“-Bemerkungen zur „schlechten Sprache“. Nach Angaben der Herausgeber:innen sind die Entwürfe auf die Zeit zwischen Ende der 1950er und Anfang der 1960er zu datieren (vgl. KS 374-377 u. 747-748), was die Vermutung nahe legt, sie seien (wenigstens zum Teil) in Zusammenhang mit dem Gulliver-Essays entstanden. Dies gilt im Besonderen für den Entwurf „Sprache der Politik und der Medien“, wo u. a. die Rede vom großen „Lauf der Dinge“ (also vom cours des choses, KS, 376) ist. 
Autorengruppe verbindet und die sie an der differenzbewussten Vielfalt der individuellen Mundarten exemplifiziert - eine Position, die an sich nicht verwunderlich ist, bedenkt man, dass das Abenteuer der Revue sich nicht zuletzt als „Abenteuer mit der Sprache“ mit ungewissen Ausgang (KS, 388) charakterisiert. ${ }^{41}$

Die explizite Verteidigung der „Provinz“ und ihrer Korrelate - „Gegend“ „Eigenart“ „Dialekt“ „Redensarten“ (KS, 382-383) - als Orte des Authentischen hat in dieser Hinsicht nichts Provinzielles an sich, sondern legitimiert sich als Opposition gegen die unheilige Allianz von Politik, Technik und Wirtschaft, der eine degradierende Verdinglichung der Sprache zum „Fertigprodukt ,Sprache““ beziehungsweise „Wunschprodukt ,Sprache““ (KS, 388) entspricht. Gemeint ist der rein funktionale Wesenzug des „Esperanto“, das hier als Paradigma einer befremdlichen Tilgung der kulturellen Differenzen und singulären Identitäten anvisiert wird.

Dabei paart sich die spezifisch österreichische Genealogie der Sprachskepsis, aus der sich Bachmanns Standpunkt speist, mit dem dezidierten Bekenntnis zu einer literarischen Sprachutopie, die einige Affinitäten mit den Positionen der italienischen Gruppe (insbesondere mit Pasolini und Calvino) aufweist und in die der Essay dann mündet. ${ }^{42}$ Hier profiliert sich die elliptische Hinwendung zum Postulat der Unübersetzbarkeit als ein Akt der Resilienz gegen die artifizielle Nivellierung der Sprache bzw. gegen die gedanken- und reibungslose Austauschbarkeit von Kulturen, Idiomen, Identitäten, die gleichbedeutend ist mit dem Verzicht auf die dialogische Kategorie der „Aufmerksamkeit“ (KS, 386) - und damit auf die Mühe der Suche nach Worten, worin der Sinn der Literatur (und hier nicht zuletzt des Dialekts als Figur einer poetischen Weltsprache) doch intrinsisch besteht.

Dann soll es, in unsrem Beruf, ruhig einmal Mühe kosten, für ein Adjektiv tagelang ein entsprechendes Adjektiv zu suchen in der anderen Sprache. Und dann wird auch nichts verloren sein, wenn einmal für ein Wort kein entsprechendes Wort gefunden wird. Wenn das Vertrauen nur da ist in den Dialekt, in das, was übersetzbar an ihm ist, in das, was unübersetzbar bleibt. (KS, 395)

41 Unter dem Titel Cours des Choses/Sur la traduction widmet Blanchot einen ganzen Absatz seiner programmatischen Thesen zum Thema Übersetzung, indem er zum einen das Problem der „langue dialectale“ stellt, zum anderen eine philosophische Typisierung des Übersetzers als „home nostalgique“ andeutet. Vgl. Blanchot: Textes préparatoires, in: Lignes (wie Anm. 18), 179-191, hier: 187. Ein Ort des Authentischen ist die Provinz - bei allen anderen ästhetischen und literaturpolitischen Unterschieden - auch für Grass (vgl. Anm. 29).

42 Freilich stehen Calvino und Pasolini innerhalb der italienischen Literatur für zwei jeweils sehr differenzierte Standpunkte. So auch im Falle der Debatte über die Transformationen der italienischen Sprache (,italiano tecnologico“ und/oder „antilingua“) im Zuge der beschleunigten Industrialisierung des Landes der 1950er und 1960er Jahre. Reflexe dieser Debatte sind unter anderem sowohl im Gulliver-Heft als auch in der Zeitschrift il menabò dokumentiert und sind insofern als indirekte Referenzpunkte auch für Bachmanns Positionen zu betrachten. Vgl. dazu Italo Calvino: L'antilingua, in: ders.: Una pietra sopra. Discorsi di letteratura e società, Torino 1980, 122-126; Pier Paolo Pasolini: Diario lingustico, in: ders: Empirismo eretico, Milano 1972, 36-48. 


\section{„[...] historisch denken und vor allem utopisch denken“: Berlin - Prag, hin und zurück}

Repräsentiert der Berlin-Komplex den neuralgischen Kern der Europa-Reflexionen der Gulliver-Gruppe, so dürfte es kaum verwundern, dass Bachmann ihn zum Stoff ihrer Dankesrede zum Georg-Büchner-Preis macht..$^{43}$ Allerdings treten zu dieser zugleich ästhetischen und politischen Entscheidung noch weitere Faktoren hinzu, unter denen sicherlich der besondere Standpunkt der Dichterin im West-Berlin des Jahres 1964 eine erstrangige Motivation darstellt. „Richtig“ scheint nämlich der Dichterin die Anknüpfung an ihre direkte Erfahrung, mit anderen Worten

in Deutschland über Berlin zu sprechen, nachdem ich dort eineinhalb Jahre verbracht habe, an einem gestörten Ort, in einer Verstörung, die von diesen Störungen einiges aufzunehmen fähig war. (GuI, 49) ${ }^{44}$

Das im Nachlass erhaltene Palimpsest der verschiedenen Überarbeitungsstufen der Rede mit den verschiedenen progressiven Streichungen, Ergänzungen, wiederholten Korrekturgängen und Kurswechseln zeugt von einer langwierigen Entstehung sowohl des literarischen Texts als auch der nicht weniger durchdachten poetologischen Einführung. Im Hinblick auf die Situation ihrer Ansprache im Akademiesaal geht es Bachmann eindeutig darum, die eigene Sprechposition klar zu fixieren, um dann ebenso unmissverständlich die eigene Rolle als Außenstehende und insofern als Beobachterin fremder Zustände zu profilieren.

$\mathrm{Zu}$ dieser programmatischen Haltung gehört weiterhin die erkennbare Intention, eine „deutsche“ Rede zu halten, die als solche definiert und gedeutet werden muss. Stark und unmittelbar soll folglich der Bezug auf die konkrete Wirklichkeit wirken, um bezüglich des Ausnahmezustands der Stadt Berlin Auswege ins Abstrakte, sei es auch nur als Alibi der Imagination, zu verhindern. (TKA I, 228-232) „Ihre Mauer ist eine Mauer von Kafka, sie ist rascher ein Symbol, ein Mythos, als eine Realität aus Beton und Stacheldraht“ (KS, 378), notiert Bachmann etwa bereits in Zusammenhang mit dem Gulliver-Tagebuch.

Die Chiffren „Krankheit“ und „Tod“, die bereits im ursprünglichem Prosa-Entwurf Sterben für Berlin eine zentrale Rolle spielen, werden hier wieder aufgegriffen

43, „[...] historisch denken und vor allem utopisch denken“ (KS, 388).

44 Auf werkgeschichtlicher Ebene ist auf die Berliner Zeit auch die Entstehungsphase des TodesartenProjekts zu datieren. Vgl. dazu insbesondere Else Schlinsog: Berliner Zufälle. Ingeborg Bachmanns Todesarten-Projekt, Würzburg 2005. Trotz der erklärten Aversion gegen klischeehafte Verallgemeinerungen (KS, 399) sind Bachmanns rekurrierende feindliche Bemerkungen über „die Deutschen“, die als solche - mitunter als implizite symbolische Gesprächspartner („Ihr geliebten verdammten Deutschen“ KS, 379) - in ihren Berliner Notizen immer wieder vorkommen, kaum zu übersehen. 
und politisch weiter transformiert. Gleichzeitig wird der von der Akademie nahelegte Rückbezug auf Büchners Werk im Sinne einer Reverenz zum kongenialen Anlass, die Erwartungen des Auditoriums grundsätzlich zu subvertieren und an die schizophrene Krankheit Lenz' - der bekannte Protagonist der gleichnamigen Novelle - als tragendes Motiv des Prosaentwurfs anzuknüpfen.

Aus dieser Prämisse ergibt sich ein intertextueller Kurzschluss - nämlich die Korrelation zwischen dem Riss in der Seele von Lenz, der durch Büchners medizinischen Terminus „Zufälle“ evoziert wird, und der gespaltenen Mauerstadt - eine signifikante Engführung, die den Weg bahnt, um die Psychopathologie des Berliner Alltags in den Mittelpunkt der „Darstellung“ zu rücken.

\begin{abstract}
Wovon reden? Von etwas Naheliegendem am besten. [...] Es wird von einer Gegend hier die Rede sein, umständehalber, von einer Stadt, da mehr sich nicht anbietet, einer die sich auf „Teilung“ hinausreden möchte. Teilung: das ist ein anderes Wort, es nimmt vieles ab, das Denken nicht zuletzt. Es hört sich an nach Operation; postoperative Schmerzen nicht ausgeschlossen, letaler Ausgang selten. Es muß also, wenn es um Zufälle geht, etwas weit zurückliegen, intermittieren, konsequent aber wiederkommen mit neuen Zufällen. (TKA I, 228-231) ${ }^{45}$
\end{abstract}

Es ist also nicht nur die latente Geste der Empörung, die Bachmann ihren Zuhörern zumutet, sondern auch der formale Radikalismus des Prosaduktus, in dem Kriegsmanöver, verstörte Gegenwart und verbrecherische Vergangenheit in eins fallen und durch das strukturierende Prinzip des Fragments ineinanderfließen - eine singuläre ,Chronik der Zeit‘, die sich neben den beiden anderen von Blanchot und Johnson durch eine dezidierte Hinwendung zum konkreten Geschehen bewusst positioniert.

So still ist es geworden und Nacht. Seit damals war niemand mehr auf der Straße. Versandet und verwachsen sind die alten Villen, sinken immer tiefer ein in den Gärten. Am Knie der Königsallee fallen, jetzt ganz gedämpft, die Schüsse auf Rathenau. In Plötzensee wird gehenkt. In der Telefonzelle rollen die Pfenningsstücke - alle umsonst eingeworfen - unten wieder heraus. Es kommt keine Verbindung zustande. (TKA I, 218-219)

45 Von der schockartigen Wirkung der Rede in Darmstadt zeugen etliche Quellen. Vgl. etwa die Korrespondenz des Akademiepräsidenten Hans W. Eppelsheimer, zit. in: Sigrid Weigel: Ingeborg Bachmann. Hinterlassenschaft unter Wahrung des Briefgeheimnisses, Wien 1999, 376. Ähnliches berichtet Klaus Wagenbach über eine Lesung Bachmanns vor dem Westberliner Publikum, vgl: Klaus Wagenbach (Hrsg.): Nachwort zu Ingeborg Bachmann, in: Ingeborg Bachmann: Ein Ort für Zufälle, hrsg. von Klaus Wagenbach, mit Zeichnungen von Günter Grass, Berlin 1994. Siehe auch Anna M. Parkinson: Taking Breath. The Ethical Stakes of Affect in Ingeborg Bachmanns Ein Ort für Zufälle, in: Caitríona Leahy, Bernadette Cronin (Hrsg.): Re-Acting to Ingeborg Bachmann. New Essays and Perfomances, Würzburg 2006, 65-75. 
Die endgültige Textpartitur, die in Darmstadt vorgelesen wird, besteht aus insgesamt 21 Ausschnitten, ${ }^{46}$ die sich als rhapsodische Sequenz von scheinbar willkürlich aneinandergereihten Momentaufnahmen artikulieren. Dabei suggerieren Verfahren der Verdichtung und der Verschlüsselung nicht weniger als das grammatische undifferenzierte Subjekt ,es', das durch den Text gespenstisch geistert, einen mäandernden bewussten-unbewussten Assoziationsfluss, in dem sich alles wie in einer fatalen Verkettung als selbstverschuldete „Konsequenz auf die Konsequenz“ (TKA I, 190) erweist. ${ }^{47}$ Auf diese Weise funktioniert das intermittierende Prosaarrangement als textuelle Simulation der Aufzeichnung eines Krankheitsverlaufs, wobei die Schreibweise sowohl die akuten Phasen als auch die illusorischen Beruhigungsmomente nachbildet. Insofern suggeriert die fast identische Bildfolge des ersten und letzten Textausschnitts eine verhängnisvolle Wiederholung des Gleichen in der klaustrophobischen Enge einer zirkulären Struktur.

Während Berlin eine finstere Chiffre der „zerstörten Vernunft“ Europas (KS, 395) repräsentiert, gestaltet sich die zwischen historischer Realität und poetischer Phantasie schwebende Landschaft Böhmens kontrapunktisch als Ort einer belebend grenzüberschreitenden Zirkulation von „Worten und Werten“ (KS, 378), Sprachen und Menschen..$^{48}$ Markiert die Reise nach Prag die Erfahrung einer imaginierten Heimkehr im Zeichen eines als transnational konturierten Kulturraums, so ist es interessant zu konstatieren, dass das Gedicht Böhmen liegt am Meer nach Bachmanns Vorstellung für die Zeitschrift Gulliver vorgesehen war, ${ }^{49}$ wo es ohne weiteres einen entsprechend konnotiertes Bedeutungsgefüge gefunden hätte.

46 In der Druckfassung, die 1965 mit Zeichnungen von Günter Grass beim Berliner Wagenbach-Verlag erscheint, sind es 26. Zu den Ergänzungen vgl. Christian Däufel: Ingeborg Bachmanns Ein Ort für Zufälle. Ein interpretierender Kommentar, Berlin/Boston 2013, $104 \mathrm{ff}$.

47 Zum Echo der Aktualität, die Bachmann mit der visionären Bildsprache ihrer Rede verknüpft, vgl. insbesondere Jost Schneider: Historischer Kontext und politische Implikationen der Büchnerpreisrede Ingeborg Bachmanns, in: Monika Albrecht, Dirk Göttsche (Hrsg.): „Über die Zeit schreiben.“ Zwei Literatur- und kulturwissenschaftliche Essays zum Werk Ingeborg Bachmanns, Würzburg 2000, 127-141.

48 In den Paralipomena zur „deutschen Rede“ verweist Bachmann auf die Kluft zwischen Berlin und dem Stichwort „Herkunft“: ,Etwas Land, etwas anders als hier, Tal, Stadt, Seen, österreichisch, windisch, verklemmt zwischen drei Länder, Italien, Kroatien, Ungarn, Böhmen“. (TKA I, 178) Zur Konstellation der böhmischen Reise, vgl. insbesondere Hans Höller, Arturo Larcati: Ingeborg Bachmanns Winterreise. Die Geschichte von Böhmen liegt am Meer, München/Berlin/Zürich 2016.

49 Vgl. den Brief an Hans Paeschke vom 23.01.1966 zit. in: Daniel Graf: Wiederkehr und Antithese. Zyklische Komposition in der Lyrik Ingeborg Bachmanns, Heidelberg 2011, 160-161. Bekanntlich wurde das Gedicht erst im November 1968 als Teil des kleinen Zyklus Vier Gedichte (zusammen mit Enigma, Prag Jänner 1964, Keine Delikatessen) in der Zeitschrift Kursbuch gedruckt, nachdem im August die Hoffnungen des Prager Frühlings durch den Einmarsch der sowjetischen Panzer zerschlagen worden waren. 
Hinsichtlich der kompositorischen Strategie wird die Architektur der Verse durch das strukturierende zentrale Motiv des „Angrenzens“ orchestriert, das nicht nur eine eigene semantische Valenz besitzt, sondern auch als formalästhetisches Gesetz die Logik der Lyrik bestimmt.

Sind hierorts Häuser grün, tret ich noch in ein Haus.

Sind hier die Brücken heil, geh ich auf gutem Grund.

Ist Liebesmüh in alle Zeit verloren, verlier ich sie hier gern.

Bin ich's nicht, ist es einer, der ist so gut wie ich.

Grenzt hier ein Wort an mich, so laß ich's grenzen.

Liegt Böhmen noch am Meer, glaub ich den Meeren wieder.

Und glaub ich noch ans Meer, so hoffe ich auf Land.

Bin ich's, so ists ein jeder, der ist soviel wie ich.

Ich will nichts mehr für mich. Ich will zugrunde gehn.

Zugrund - das heißt zum Meer, dort find ich Böhmen wieder.

Zugrund gerichtet, wach ich ruhig auf.

Von Grund auf weiß ich jetzt, und ich bin unverloren. (W I, 167)

Die lyrische Plausibilität von Böhmen am Meer geht hier Hand in Hand mit der heilenden Rückprojektion auf die Dimension einer vertrauten Zugehörigkeit, wobei die Konstellation der Meer-Grenze mit einem animierenden Moment der Destabilisierung im Zeichen von Durchlässigkeit und Hybridisierung assoziiert wird. So kann auch die Figur des Schiffbruchs zu einer produktiven Chance verwandelt werden - dafür steht die poetische Intuition des „unverloren“ -, in der Gefahr und Rettung in eins fallen.

Kommt her, ihr Böhmen alle, Seefahrer, Hafenhuren und Schiffe unverankert. Wollt ihr nicht böhmisch sein, Illyrer, Veroneser, und Venezianer alle. [...]

\section{$[\ldots]$}

Ich grenz noch an ein Wort und an ein andres Land, ich grenz, wie wenig auch, an alles immer mehr,

ein Böhme, ein Vagant, der nichts hat, den nichts hält, begabt nur noch, vom Meer, das strittig ist, Land meiner Wahl zu sehen. (W I, 167-168)

Ferner wirkt sich das Motiv des „Angrenzens“ in der Struktur der Konditionalsätze aus, die durch die Inversion der Verben indirekt vermittelt und zugleich verkürzt werden, wobei die Apostrophen und Vokalelisionen die Funktion von evozierenden Signalen der dialektalen Färbung der Austriazismen übernehmen. 
Mit seinem magisch beschwörenden Resonanzraum vermag das Denkbild Böhmen das Sediment einer Ursprache zu chiffrieren, in dem konvergierende Sinnkonstellationen mitschwingen - zum einen ein heimatliches Alphabet der Seele, zum anderen die verborgenen Spuren einer imaginiert polyphonen Herkunfts- und Sprachlandschaft, die sich simultan als Natur- und Geschichtsraum behauptet und sich mit dem positiv besetzten Paradigma „Haus Österreich“ (GuI, 78) identifiziert. ${ }^{50}$

Als mitteleuropäischer Kosmos der kulturellen und transnationalen Diversitäten vermag es noch in dieser Hinsicht, zumal vor dem Hintergrund der zeitkritischen Utopie von Gulliver, ein eigenes subversives Potential zu beanspruchen, insofern es sich als poetisch-politische Aussage gegen die Spaltung Europas richtet. ${ }^{51}$

Zwei Jahre vor dem Mauerfall wird Enzensberger seine Hommage an die Dichterin Bachmann mit einer gezielten Referenz an Böhmen liegt am Meer verbinden, das Gedicht, das sie selbst bekanntlich für ihr bestes hielt. ${ }^{52}$ Enzensbergers ,futuristischer Fiktion zufolge kommt im Jahre 2006 ein amerikanischer Journalist nach Europa - die Mauer ist zwar gefallen, der Lauf der Dinge ist aber trotzdem nicht so erfreulich. In Prag, letzte Station vor der Abreise, kommt der Amerikaner in Dialog mit einem jungen Taxifahrer, der ihm als enthusiastischer Literaturstudent jene grenzüberschreitende lyrische Phantasie - Böhmen am Meer - empfiehlt. Auf die verdutzte Reaktion des fremden Besuchers sorgt die ironische Replik des jungen Einheimischen für eine weitere allusive Akzentsetzung: ,und sollten Sie nicht alles verstanden haben, machen Sie sich nichts daraus [...] das liegt nicht an Ihnen [...] es liegt ganz einfach an Europa“. ${ }^{53}$

50 Ingeborg Bachmann: Ein Tag wird kommen. Gespräche in Rom. Ein Porträt von Gerda Haller. Mit einem Nachwort von Hans Höller, Salzburg/Wien, 61-82, hier: 80 f. Zur politisch-utopischen Motivkonstellation Österreich/Böhmen vgl. u.a. Jürgen Wertheimer: Ingeborg Bachmanns skeptischer Blick auf Europa, in: ders. (Hrsg.): Suchbild Europa. Künstlerische Konzepte der Moderne, Amsterdam/Atlanta, GA 1995, 38-45; Christine Ivanovic: Böhmen als Heterotopie, in: Mathias Meyer (Hrsg.): Werke von Ingeborg Bachmann. Interpretationen, Stuttgart 2002, 109-121; Judith Kasper: Boemia. Ingeborg Bachmann, in: dies.: Trauma e nostalgia. Per una lettura del concetto di Heimat, Genova 2009, 153-175; Katya Krylova: Österreichthematik, in: Albrecht, Göttsche (Anm. 6), 289-294 sowie Dirk Weissmann: Mehrsprachigkeit, in: ebd., 404-408.

51 Zur kulturgeschichtlichen Konnotation von den beiden Termini „Mitteleuropa“ / „Zentraleuropa“ vgl. inbesondere: Moritz Czázy: Das Gedächtnis Zentraleuropas. Kulturelle und literarische Projektionen auf eine Region, Köln/Weimar/Wien 2019; Yvonne Zivkovic: Ingeborg Bachmann and Peter Handke. The Austrian Periphery and Mitteleuropa, in: dies.: The Literary Politics of Mitteleuropa. Reconfiguring Spatial Memory in Austrian and Yugoslav Literature after 1945, Rochester, NY 2021, 81-162.

52 Bachmann (Anm. 50), $80 \mathrm{f}$.

53 Hans Magnus Enzensberger: Böhmen am Meer. Ein Hörspiel aus dem Jahr 2006, in: ders.: Der fliegende Robert. Gedichte, Szenen, Essays, Frankfurt a. M. 1989, 126-170, hier: 170. 
